\title{
Correspondence Prepulse Inhibition Deficits in Obsessive-Compulsive Disorder are More Pronounced in Females
}

\author{
Susanne E Ahmari*, I, Victoria B Risbrough², Mark A Geyer ${ }^{2}$ and H Blair Simpson ${ }^{3,4}$ \\ 'Department of Psychiatry and Center for Neural Basis of Cognition, University of Pittsburgh, Pittsburgh, PA, USA; ${ }^{2}$ Department of Psychiatry, \\ University of California, San Diego, CA, USA; ${ }^{3}$ Department of Psychiatry, Columbia University, College of Physicians \& Surgeons, New York, NY, USA; \\ ${ }^{4}$ Division of Clinical Therapeutics, New York State Psychiatric Institute, New York, NY, USA
}

Neuropsychopharmacology (2016) 4I, 2963-2964; doi: I0.1038/npp.2015.363

Obsessive-compulsive disorder (OCD) is a disabling and chronic psychiatric disorder, but despite its severity and prevalence, relatively little is known about its neurobiology. Substantial evidence suggests that abnormalities in structure and function of cortico-striato-thalamo-cortical (CSTC) circuits underlie OCD pathophysiology (Ahmari and Dougherty 2015). This fact highlights the need to identify probes of CSTC circuits that are consistently abnormal in OCD patients, in order to facilitate translational studies. In pursuit of this goal, in a previous study (Ahmari et al, 2012) we found that unmedicated adults with OCD had deficits in sensorimotor gating as measured by prepulse inhibition (PPI) of the acoustic startle response at three different prepulse intensities (74, 78, and $86 \mathrm{~dB} ; n=22$ OCD, 22 controls). Exploratory analyses suggested that OCD patients with a history of tics had lower PPI than those without tics.

Owing to recent studies highlighting potential sex differences in both OCD genetic substrates (Taylor 2013) and clinical course (Torresan et al, 2013), the known effects of hormonal state on PPI (Jovanovic et al, 2004), and increasing recognition of the importance of sex effects in psychiatric pathophysiology (see NIH NOT-OD-15-102: Consideration of Sex as a Biological Variable in NIHfunded Research; Clayton and Collins 2014), we reanalyzed our PPI data to examine males and females separately (see original manuscript for demographics and clinical details). In light of past literature demonstrating increased prevalence of tic-related OCD in males (Leckman et al, 1994), we hypothesized that male OCD patients were driving the observed PPI deficits in our study. Surprisingly, when male and female patients were analyzed separately, we found that PPI deficits were more pronounced in female patients (Females: OCD $(n=10)$ vs control $(n=10)$ : F $(1,18)=$ 4.539; $p<0.047$, Cohen's $d=0.88$; Males: OCD $(n=12) v s$ control $(n=12): \quad$ F $(1,22)=2.269 ; \quad p>0.146$, Cohen's $d=0.65$ ). This effect was even more striking when patients

\footnotetext{
* Correspondence: Dr SE Ahmari, Department of Psychiatry, Center for Neural Basis of Cognition, University of Pittsburgh, 450 Technology Drive, Room 227, Pittsburgh, PA I 2219 , USA, Tel: +4I 2624 3183, Fax: +412 624 5280, E-mail: ahmarise@upmc.edu
}

with tics ( $n=3$ males) were removed from the male sample (Males without tics: OCD vs control: F $(1,19)=0.380$; $p>0.545$, Cohen's $d=0.27$ ), and was most prominent at higher prepulse intensities (Figure 1). In females, re-analysis of timing of PPI measurements relative to menstruation revealed that although all subjects signed consent within days 1-10 of the menstrual cycle, 5 HC and 4 OCD were tested outside this window, yielding a range of cycle day 4-17 in $\mathrm{HC}$ and 1-17 in OCD (ie, follicular/ovulation phase); average menstrual cycle day was the same in both groups $(\mathrm{OCD}=$ $9.5 \pm 5.1 ; \mathrm{HC}=9.5 \pm 5.9$ ). There was no statistically significant correlation between day of menstrual cycle and PPI $\left(R^{2}: \mathrm{HC}=0.017 ; \mathrm{OCD}=0.031 ; \mathrm{F}\right.$ values: $\mathrm{HC}=0.14 ; \mathrm{OCD}=$ 0.19 ). Exploratory analyses testing the association between the consolidated PPI measure for each sex and clinical variables (OCD severity; age of OCD onset; or any of the five symptom dimensions) using Spearman's correlation indicated that females with OCD had a trend towards lower percent PPI (ie, larger deficits) with older age of OCD onset $(p<0.089)$. No other significant associations were observed in either females (all $p$-values $>0.25$ ) or males (all $p$-values $>0.33)$ with OCD.

The observed differential effect of sex suggests that pathophysiologic processes may be distinct in females and males with OCD, and that these differences may potentially be localized to the neural substrates underlying PPI, a well-established method for probing functional status of cortico-striato-pallidal circuitry in humans and rodents. Because this is a post hoc, exploratory analysis with multiple moderators in a relatively small data set, and therefore would not survive correction for multiple comparisons, these findings need to be replicated in a study in which sex effects are examined from the outset. Though the neurobiological underpinnings of this effect are unknown, the trend in females towards larger deficits when OCD onset is after typical age of menarche suggests pubertal-related circuit changes. One explanation for these changes could include a shift in the prepulse inhibition/facilitation curve towards facilitation in post-pubertal women, via increased circulating estrogen, leading to increased excitatory/inhibitory balance (Aasen et al, 2005). Dissecting the mechanisms leading to 


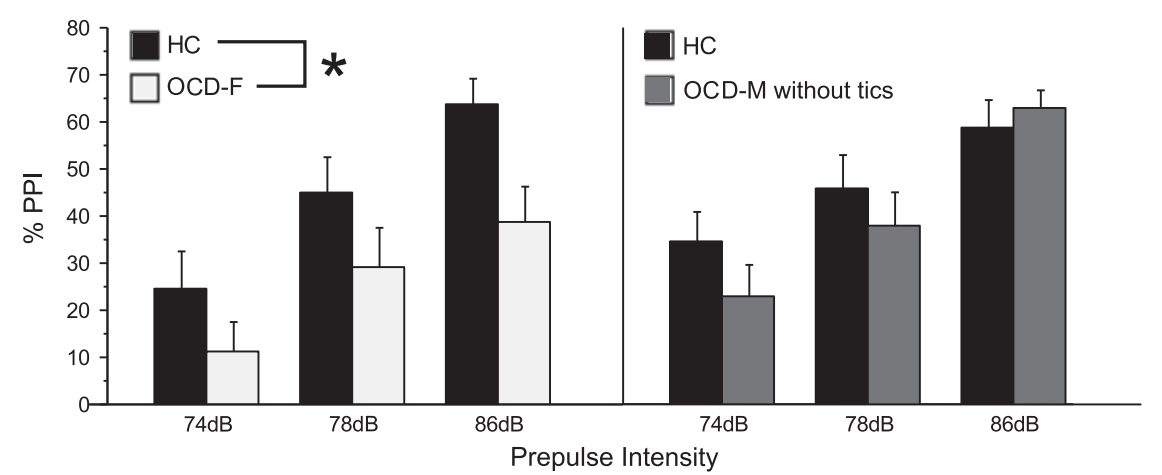

Figure I PPI deficits are more pronounced in female OCD subjects. Bar graph demonstrates percent PPI (mean \pm SEM) at 74, 78, and $86 \mathrm{~dB}$ prepulse intensities. Female OCD patients (left panel) demonstrate more pronounced PPI deficits than males, particularly when patients with a history of tics ( $n=3$ males) are removed (right panel). Females: OCD $(n=10)$ vs control $(n=10): F(I, I 8)=04.539 ; p<0.047$. Males without tics: OCD ( $n=9)$ vs control $(n=12)$ : $F(I, 19)=0.380 ; p>0.545$. HC, healthy control subjects; OCD-F, female OCD subjects; OCD-M without tics, male OCD subjects without tics. * indicates significance at $p<0.047$.

increased PPI deficits in female OCD patients could ultimately lead to discovery of targets for sex-specific, tailored OCD treatments.

\section{FUNDING AND DISCLOSURE}

In the last 3 years, Dr Simpson has received medication at no cost from Janssen Pharmaceuticals for an NIMH-funded study, consulting fees from Pfizer for advice regarding the medication Lyrica, and research funds from Neuropharm Ltd and from Transcept Pharmaceuticals to conduct clinical trials of novel medications for OCD. Dr Geyer has received consulting compensation from Abbott, Dart, Lundbeck, Neurocrine, Omeros, Otsuka, and Sunovion, and holds an equity interest in San Diego Instruments. Dr Risbrough has received consulting fees from Arena, Cenomed, and Ferring Pharmaceuticals. Dr Geyer has research grant support from NIDA, NIMH, and the US Veteran's Administration VISN 22 Mental Illness Research, Education, and Clinical Center. Dr Ahmari has no financial interests to disclose. This work was supported by an NIH T32 research fellowship, NIH K08 MH087718-01A1, and a Louis V. Gerstner, Jr Scholars Grant to S.E.A; by NIH R24 MH080022 and NIH K24 MH091555 to HBS; and by the New York State Office of Mental Health. The authors declare no conflict of interest.

\section{ACKNOWLEDGMENTS}

We would like to thank Dr James Bender, Rena Staub, and Liane Hunter for performing patient recruitment and screening; Dr Page van Meter and Dr Andrew Schmidt for database management; Kimberly Glazier for assistance with data processing; and Dr Donna Vermes, Dr Rafael Campeas, Dr Anthony Pinto, and Dr Carolyn Rodriguez for assistance with clinical ratings.

\section{REFERENCES}

Aasen I, Kolli L, Kumari V (2005). Sex effects in prepulse inhibition and facilitation of the acoustic startle response: implications for pharmacological and treatment studies. J Psychopharmacol 19: $39-45$.

Ahmari SE, Dougherty DD (2015). Dissecting OCD circuits: from animal models to targeted treatments. Depress Anxiety 32: $550-562$.

Ahmari SE, Risbrough VB, Geyer MA, Simpson HB (2012). Impaired sensorimotor gating in unmedicated adults with obsessive-compulsive disorder. Neuropsychopharmacology 37: 1216-1223.

Clayton JA, Collins FS (2014). Policy: NIH to balance sex in cell and animal studies. Nature 509: 282-283.

Jovanovic T, Szilagyi S, Chakravorty S, Fiallos AM, Lewison BJ, Parwani A et al (2004). Menstrual cycle phase effects on prepulse inhibition of acoustic startle. Psychophysiology 41: 401-406.

Leckman JF, Grice DE, Barr LC, de Vries AL, Martin C, Cohen DJ et al (1994). Tic-related vs. non-tic-related obsessive compulsive disorder. Anxiety 1: 208-215.

Taylor S (2013). Molecular genetics of obsessive-compulsive disorder: a comprehensive meta-analysis of genetic association studies. Mol Psychiatry 18: 799-805.

Torresan RC, Ramos-Cerqueira AT, Shavitt RG, do Rosário MC, de Mathis MA, Miguel EC et al (2013). Symptom dimensions, clinical course and comorbidity in men and women with obsessive-compulsive disorder. Psychiatry Res 209: 186-195. 